\title{
PC-MSDM: A quality metric for 3D point clouds
}

\author{
Gabriel Meynet, Julie Digne and Guillaume Lavoué \\ CNRS, Univ. Lyon \\ LIRIS, UMR5205, F-69622 France \\ \{gabriel.meynet, julie.digne,guillaume.lavoue\}@liris.cnrs.fr
}

\begin{abstract}
In this paper, we present PC-MSDM, an objective metric for visual quality assessment of $3 D$ point clouds. This full-reference metric is based on local curvature statistics and can be viewed as an extension for point clouds of the MSDM metric suited for 3D meshes. We evaluate its performance on an open subjective dataset of point clouds compressed by octree pruning; results show that the proposed metric outperforms its counterparts in terms of correlation with mean opinion scores.

Index Terms-Visual Quality Assessment, Point Cloud, Objective Metric.
\end{abstract}

\section{INTRODUCTION}

With the increasing capability of 3D data acquisition devices (RGBD cameras, 3D scanners), 3D graphical data are now commonplace in many applications. A popular way to represent this $3 \mathrm{D}$ content is the triangle mesh representation, which is particularly compliant with modern graphical hardware. However, many applications (e.g., telepresence systems) directly consider the raw point cloud representation produced by the acquisition devices, to avoid any complex and possibly lossy reconstruction step. A disadvantage of the point cloud representation is the size of the data which may be huge; consequently a significant amount of work has been devoted to the efficient compression of this representation [1]-[5]. This lossy compression introduces distortions that impact the perceived quality of the data. Accurate objective quality metrics are thus needed to evaluate this visual quality.

In this context, we propose a full-reference objective quality metric for 3D point clouds (without color information). It is built as an extension of the MSDM metric [6], [7] that has been designed for 3D meshes. The proposed metric takes as inputs two point clouds (the original one and the impaired one), first establishes their correspondence, then computes Gaussianweighted curvature statistics on a set of local neighbourhoods and finally produces a global score that predicts the level of impairment.

\section{RELATED WORK}

Image metrics. In the field of $2 \mathrm{D}$ image processing, the research on objective quality assessment metrics is highly developed [8]. Bottom-up approaches aim at imitating the low-level mechanisms of the human visual system (HVS), such as the Visible Difference Predictor [9]. They have been extended to cover more modern imaging format such as HDR-VDP-2 [10] for High Dynamic Range content. Top-down approaches do not rely upon the visual system principles but instead propose some signal fidelity criteria expected to correlate well with the perceptual quality such as SSIM [11] and its derivatives. A large number of top-down image quality metrics have been proposed; as for bottom-up models, they have been recently extended to new imaging format such as HDR-VQM [12].

3D mesh metrics. Inspired by image quality metrics, several perceptually-motivated metrics have been designed for 3D data represented by triangulated surfaces. They attempt to predict the visual fidelity of a distorted 3D mesh with respect to a reference one [6], [7], [13], [14]. They are mostly top-down approaches that rely on different geometric attributes (e.g., dihedral angles, curvatures). In particular, the MSDM metric [6] and its multi-scale extension MSDM2 [7] rely on curvature statistics.

3D point cloud metrics. The research on objective quality assessment of point clouds is still in its early stage. Beyond the classical point-to-point distance, several researchers have recently proposed metrics based on the projected error along the normal of the reference point [15] or the angle between the tangent planes from the points [16].

\section{THE PROPOSED METRIC}

\section{A. Overview}

Our approach is inspired by the SSIM metric of Wang et al. [11] suited for 2D images. This top-down approach considers that the structural information of the image is a relevant feature for quality assessment. As SSIM, our metric relies on differences of structure (captured via curvature statistics) computed on local corresponding neighbourhoods between the reference and distorted point clouds. Our approach is an adaptation for point clouds, of the MSDM metric designed for 3D meshes [6], [7]. For given distorted $P C_{\text {dist }}$ and reference $P C_{r e f}$ point clouds, our approach is composed of the following steps:

- Curvature computation (see Sec. III-B).

- Correspondence computation between 3D points from $P C_{\text {dist }}$ and $P C_{\text {ref }}$ (see Sec. III-C).

- Computation of local and global distortion scores (see Sec. III-D).

\section{B. Curvature computation}

To capture the local structural information of each 3D point cloud, we locally approximate the surface subtended by the point cloud and compute the surface curvature, more precisely 
the mean curvature.

To estimate the mean curvature at a point $p$, we proceed by local least squares fitting of a quadric surface. First we estimate an approximate tangent plane using Principal Component Analysis, which gives us an orthonormal frame $\left(u_{x}, u_{y}, u_{z}\right)$ such that $u_{z}$ is aligned with an approximate normal to the surface [17]. We take $p$ as the origin of the coordinate system. In this local frame, the neighbour $p_{i}$ of $p$ has coordinates $\left(x_{i}, y_{i}, z_{i}\right)$. We thus look for the quadric surface $Q(x, y)=a x^{2}+b y^{2}+c x y+d x+e y+f$ minimizing:

$$
\sum_{i}\left\|z_{i}-Q\left(x_{i}, y_{i}\right)\right\|^{2}
$$

The mean curvature can then be directly estimated from the derivatives of $Q$ that are expressed easily by its coefficients:

$$
\operatorname{Curv}(p)=\frac{\left(1+d^{2}\right) a+\left(1+e^{2}\right) b-4 a b c}{\left(1+e^{2}+d^{2}\right)^{\frac{3}{2}}}
$$

Note that the neighbourhood of $p$ is computed using a knearest neighbours algorithm, relying on a kd-tree [18].

\section{Correspondence between point clouds}

The objective is now to find, for each point $p$ from $P C_{d i s t}$, its corresponding point $\hat{p}$ on $P C_{r e f}$. Note that just considering the closest point to $p$ from $P C_{r e f}$ would lead to an inaccurate correspondence. Instead we search, for each $p$, the projection $\hat{p}$ on the $3 \mathrm{D}$ shape subtended by $P C_{r e f}$. For doing so we consider the same local quadric fitting as described above, then $\hat{p}$ is given as the projection of $p$ on the fitted quadric surface.

\section{Distortion measure}

As discussed above, our metric is mostly inspired by the SSIM image metric from Wang et al. [11]. The SSIM index computes luminance statistics on $11 \times 11$ circular neighbourhoods around each pixel. In our case, we apply the same strategy: we consider a spherical neighbourhood around each point $p$ of $P C_{d i s t}$ and we compute differences of curvature statistics between the set of point from the neighbourhood of $p$ and their corresponding 3D points on $P C_{r e f}$. For a given scale $h$, we define the neighbourhood $N(p, h)$ at each point $p$ as the set of points belonging to the sphere with center $p$ and radius $h$.

Functions $L, C$ and $S$ have already been adapted for 3D mesh quality assessment in the MSDM and MSDM2 metrics [6], [7]; for each point $p$ of $P C_{d i s t}$, we consider the following functions, defined for a scale $h$ :

$$
\begin{array}{r}
L(p)=\frac{\left\|\mu_{p}-\mu_{\hat{p}}\right\|}{\max \left(\mu_{p}, \mu_{\hat{p}}\right)+K} \\
C(p)=\frac{\left\|\sigma_{p}-\sigma_{\hat{p}}\right\|}{\max \left(\sigma_{p}, \sigma_{\hat{p}}\right)+K} \\
S(p)=\frac{\left\|\sigma_{p} \sigma_{\hat{p}}-\sigma_{p \hat{p}}\right\|}{\sigma_{p} \sigma_{\hat{p}}+K}
\end{array}
$$

where $K$ is a constant to avoid instability when denominators are close to zero. $\mu_{p}, \mu_{\hat{p}}$ are respectively Gaussian-weighted averages of curvature over the $3 \mathrm{D}$ points belonging to the $h$-scale neighbourhood of $p$ and over their projections onto $P C_{\text {ref }}$. Similarly $\sigma_{p}, \sigma_{\hat{p}}$ and $\sigma_{p \hat{p}}$ are Gaussian-weighted standard deviations and covariance of curvature. Our local distortion measure is then defined, for each point $p$ from $P C_{\text {dist }}$ as follows:

$$
L D(p)=\frac{\alpha L(p)+\beta C(p)+\gamma S(p)}{\alpha+\beta+\gamma}
$$

$\alpha, \beta$ and $\gamma$ were set respectively to 1,1 and 0.5 as in [6], [7]

These local distortion scores can then be combined into a single score that assesses the global visual distortion. In the original 2D metric from Wang et al. [11], a simple sum over the pixels is calculated. In our case, we compute a Minkowski pooling defined as follows:

$$
P C-M S D M_{\mathcal{P C}_{\text {dist }} \rightarrow \mathcal{P C} \mathcal{C}_{\text {ref }}}=\left(\frac{1}{\left|\mathcal{P} \mathcal{C}_{\text {dist }}\right|} \sum_{p \in \mathcal{P} \mathcal{C}_{\text {dist }}} L D(p)^{n}\right)^{\frac{1}{n}}
$$

As $n$ increases, more emphasis is given to the high distortion regions; in our metric, we consider $n=2$.

$P C$ - $M S D M_{\mathcal{P C}} \mathcal{C}_{\text {dist }} \rightarrow \mathcal{P C} \mathcal{C}_{\text {ref }}$ evaluates the structural distortion of the distorted model regarding the reference one. In order to strengthen the robustness of our method and to obtain a symmetric measure we also compute $P C-M S D M_{\mathcal{P C}} \mathcal{C}_{r e f} \rightarrow \mathcal{P C}_{\text {dist }}$ and we retain the average as our final distortion measure $P C$-MSDM. This metric is within the range $[0,1]$, a value of 0 means that the two point clouds are identical while values near 1 mean that they are visually very different.

\section{EXPERIMENTS}

\section{A. Parameters of the metric}

The two main parameters of our metric are (1) the number $k$ of neighbours used to compute the quadric fitting for curvature computation (see Sec. III-B) and correspondence (see Sec. III-C), and (2) the scale $h$ used to compute curvature statistics (see Sec. III-D). Both parameters actually define the scale of the distortion that we want to capture and are linked to the point cloud resolutions. In our experiment we consider $k=5$ and $h=0.02 \times B B$ with $B B$ the bounding box length.

\section{B. Performance evaluation}

A quality assessment metric is typically evaluated by computing its correlation with the human judgement, i.e. mean opinion scores (MOS) from distorted stimuli obtained through a subjective experiment. To the best of our knowledge, these exist only one publicly-available subject-rated database of 3D point clouds [19] ${ }^{1}$. This database was originally created at the Multimedia Signal Processing Group from EPFL [19] and further enriched [20]. It contains 30 point clouds between $9 \mathrm{~K}$ and $35 \mathrm{~K}$ points generated from 6 references. The reference models have been compressed by octree pruning using four different levels so as to keep resp. 30\%, 50\%, $70 \%$ and $90 \%$ of the points. This dataset has been and evaluated by five different labs and thus is associated with five sets of mean opinion scores. Note that the point clouds were visualized as 
surfaces after a mesh reconstruction process.

To evaluate the performance of our metric PC-MSDM, we compute the correlation between its computed values and the mean opinion scores of the point clouds of the dataset; we consider the Spearman rank order correlation and the Pearson linear correlation coefficients computed after performing a nonlinear regression on the metric values using a logistic function. Figure 1 provide correlation results, averaged over the five datasets (with the standard deviations). We also provide a comparison with several existing point clouds metrics of which results are provided in [20]. Note that, as in [20], we apply our metric both after and be fore surface mesh reconstruction (in the latter case we also provide results of MSDM2, which applies only on surface meshes). Results show that our metric significantly outperforms its counterparts. Moreover, even if the surface information is lost, it attains the performance of MSDM2 while having the benefit of being applicable directly on point clouds, without mesh reconstruction process.

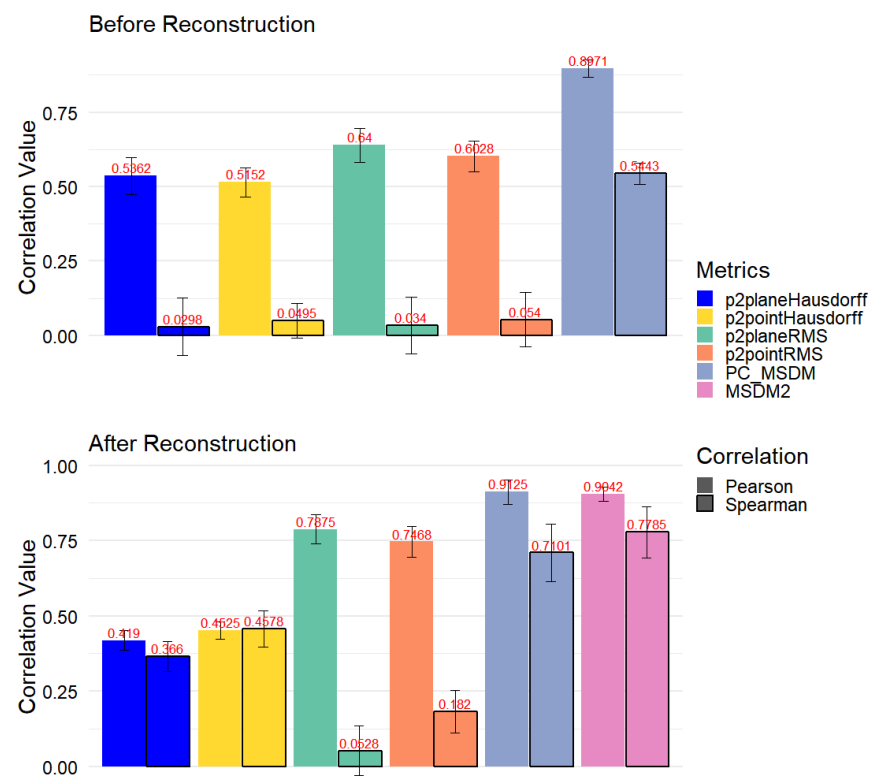

Fig. 1. Performance of point cloud metrics on the dataset from [20], considering point clouds before (top) and after (bottom) surface reconstruction. Bars without border represent Pearson correlations and bars with black borders represent Spearman correlations.

\section{CONCLUSION}

We have presented a full reference metric for the quality assessment of 3D point clouds. The proposed local analysis of curvature demonstrates a higher capability of quality prediction than pure geometric distance. Note that the proposed metric will be made publicly available online. Future work includes a deeper study of the influence of scale as well as the integration of colors.

\section{ACKNOWLEDGMENT}

We are grateful to Antonio Pinheiro and Evangelos Alexiou for providing the subjective dataset. This work was supported by French National Research Agency as part of ANR-PISCo project (ANR-17-CE33-0005).

\section{REFERENCES}

[1] D. Thanou, P. A. Chou, and P. Frossard, "Graph-based compression of dynamic 3D point cloud sequences," IEEE Transactions on Image Processing, vol. 25, no. 4, pp. 1765-1778, 2016.

[2] Y. Huang, J. Peng, C. C. Kuo, and M. Gopi, "A generic scheme for progressive point cloud coding," IEEE Transactions on Visualization and Computer Graphics, vol. 14, no. 2, pp. 440-453, 2008.

[3] R. De Queiroz and P. A. Chou, "Transform Coding for Point Clouds Using a Gaussian Process Model," IEEE Transactions on Image Processing, vol. 7149, 2017.

[4] R. Mekuria, K. Blom, and P. Cesar, "Design, Implementation, and Evaluation of a Point Cloud Codec for Tele-Immersive Video," IEEE Transactions on Circuits and Systems for Video Technology, vol. 27, no. 4, pp. 828-842, 2017.

[5] S. Schwarz, M. Preda, V. Baroncini, M. Budagavi, P. Cesar, P. A. Chou, R. A. Cohen, M. Krivokuca, S. Lasserre, Z. Li, J. Llach, K. Mammou, R. Mekuria, O. Nakagami, E. Siahaan, A. Tabatabai, A. M. Tourapis, and V. Zakharchenko, "Emerging MPEG Standards for Point Cloud Compression," IEEE Journal on Emerging and Selected Topics in Circuits and Systems, vol. 9, no. 1, pp. 133-148, 2019.

[6] G. Lavoue, E. Drelie Gelasca, F. Dupont, A. Baskurt, and T. Ebrahimi, "Perceptually driven 3D distance metrics with application to watermarking," in SPIE, vol. 6312, no. 1. SPIE, aug 2006.

[7] G. Lavoué, "A Multiscale Metric for 3D Mesh Visual Quality Assessment," Computer Graphics Forum, vol. 30, no. 5, pp. 1427-1437, 2011.

[8] Z. Wang and A. C. Bovik, Modern Image Quality Assessment. Morgan \& Claypool Publishers, jan 2006, vol. 2, no. 1.

[9] S. Daly, "The visible differences predictor: an algorithm for the assessment of image fidelity," in Digital images and human vision, Andrew B. Watson, Ed. Cambridge: MIT Press, oct 1993, pp. 179-206.

[10] R. Mantiuk, K. J. Kim, A. G. Rempel, and W. Heidrich, "HDR-VDP-2 : A calibrated visual metric for visibility and quality predictions in all luminance conditions," ACM Siggraph, 2011.

[11] Z. Wang, A. Bovik, H. Sheikh, and E. Simoncelli, "Image quality assessment: From error visibility to structural similarity," IEEE Transactions on Image Processing, vol. 13, no. 4, pp. 600-612, 2004.

[12] M. Narwaria, M. Perreira, P. L. Callet, M. Narwaria, M. Perreira, D. Silva, and P. L. Callet, "HDR-VQM : An Objective Quality Measure for High Dynamic Range Video," Signal Processing: Image Communication, vol. 35, pp. 46-60, 2015.

[13] M. Corsini, E. D. Gelasca, T. Ebrahimi, and M. Barni, "Watermarked 3D Mesh Quality Assessment," IEEE Transactions on Multimedia, vol. 9, no. 2, pp. 247-256, feb 2007.

[14] L. Váša and J. Rus, "Dihedral Angle Mesh Error: a fast perception correlated distortion measure for fixed connectivity triangle meshes," Computer Graphics Forum, vol. 31, no. 5, 2012.

[15] D. Tian, H. Ochimizu, C. Feng, R. Cohen, and A. Vetro, "Geometric distortion metrics for point cloud compression," ICIP, pp. 3460-3464, 2018.

[16] E. Alexiou and T. Ebrahimi, "Point Cloud Quality Assessment Metric Based on Angular Similarity," ICME, pp. 1-6, 2018.

[17] N. Mitra and A. Nguyen, "Estimating Surface Normals in Noisy Point Cloud Data," in Symposium on Computational geometry, 2003.

[18] J. L. Blanco and P. K. Rai, "nanoflann: a C++ header-only fork of FLANN, a library for nearest neighbor (NN) with kd-trees," https://github.com/jlblancoc/nanoflann, 2014.

[19] T. Ebrahimi and E. Alexiou, "On the performance of metrics to predict quality in point cloud representations," Applications of Digital Image Processing XL, no. c, p. 53, 2017.

[20] E. Alexiou, M. V. Bernardo, A. Luis, S. Cruz, and L. G. Dmitrovi, "Point cloud subjective evaluation methodology based on 2D rendering," International Conference on Quality of Multimedia Experience, 2018.

\footnotetext{
${ }^{1}$ https://mmspg.epfl.ch/reconstructed-point-clouds-results
} 